\title{
The odd inverse Pareto-G class: properties and applications
}

\author{
Maha A. Aldahlan ${ }^{a, b}$, Ahmed Z. Afify, ${ }^{c, *}$ A-Hadi N. Ahmed ${ }^{d}$ \\ a Statistics Department, Faculty of Science, King Abdulaziz University, Jeddah, KSA. \\ ${ }^{b}$ Statistics Department, Faculty of Science, University of Jeddah, KSA. \\ ${ }^{c}$ Department of Statistics, Mathematics and Insurance, Benha University, Egypt. \\ ${ }^{d}$ Department of mathematical statistics, ISSR, Cairo University, Egypt.
}

\begin{abstract}
We introduce a new family of continuous distributions called the odd inverse Pareto-G class which extends the exponentiatedG family due to Gupta et al. [R. C. Gupta, P. L. Gupta, R. D. Gupta, Comm. Statist. Theory Methods, 27 (1998), 887-904] and the Marshall-Olkin-G class due to Marshall and Olkin [A. W. Marshall, I. Olkin, Biometrika, 84 (1997), 641-652]. We define and study two special models of the proposed family which are capable of modeling various shapes of aging and failure criteria. The special models of this family can provide reversed J-shape, symmetric, left skewed, right skewed, unimodal or bimodal shapes for the density function. Some of its mathematical properties are derived. The maximum likelihood method is used to estimate the model parameters. By means of four real data sets we show that the special models of this family have superior performance over several existing distributions.
\end{abstract}

Keywords: Generating function, inverse Pareto distribution, maximum likelihood, order statistic, Rényi entropy. 2010 MSC: 62E10, 60E05.

(C)2019 All rights reserved.

\section{Introduction}

The procedure of expanding a family of distributions by adding new shape parameters is well-known in the statistical literature. Recently, various families of distributions have been constructed by extending some well-known lifetime distributions by adding one or more shape parameter(s). The role of the extra shape parameters is to introduce skewness and to vary tail weights and provide more flexibility to model both monotonic and non-monotonic failure rates even though the baseline failure rate may be monotonic.

The beta, gamma, Kumaraswamy, logistic, Lomax, Burr X, Weibull, Lindley, Gompertz, Poisson, exponentiated Weibull, and Burr XII distributions, among others, are used to construct the beta-G (Eugene et al., [14]), gamma-G (Zografos and Balakrishnan, [33]), Kumaraswamy-G (Cordeiro and de Castro, [11]), logistic-G (Torabi and Montazeri, [31]), Lomax-G (Cordeiro et al., [13]), Weibull-G (Bourguignon et al., [8]), exponentiated Weibull-H (Cordeiro et al., [11]) and odd Lomax-G (Cordeiro et al., [9]) families.

\footnotetext{
*Corresponding author

Email addresses: maldahlan@kau.edu.sa (Maha A. Aldahlan), ahmed.afify@fcom.bu.edu.eg (Ahmed Z. Afify),

drhadi@cu.edu.eg (A-Hadi N. Ahmed)
}

doi: $10.22436 /$ jnsa.012.05.02

Received: 2018-07-05 Revised: 2018-10-05 Accepted: 2018-10-26 
To the best of our knowledge the inverse Pareto (IP) distribution will be used for the first time to propose a new wider family of distributions to fill in the gab in the literature. The two-parameter IP distribution (Klugman et al. [19, P. 707, Sec. A.2.3.2]) has the cumulative distribution function (CDF) given (for $x>0$ ) by

$$
G(x ; \alpha, \beta)=\left(\frac{x}{\beta+x}\right)^{\alpha}
$$

where $\alpha$ and $\beta$ are positive shape parameters.

The probability density function (PDF) of the IP distribution is given by

$$
g(x ; \alpha, \beta)=\frac{\alpha \beta x^{\alpha-1}}{(\beta+x)^{\alpha+1}} .
$$

In this paper, a new class of distributions called the odd inverse Pareto-G (OIP-G) family is constructed based on the IP distribution and the T-X family (Alzaatreh et al., [5]). We study some of its special models and provide a comprehensive description of some of its mathematical properties.

Let $\mathrm{G}(\chi ; \varphi)$ be the baseline CDF with parameter vector $\varphi$. Then, the CDF and PDF (for $\chi>0$ ) of the OIP-G family are defined by

$$
F(x ; \alpha, \beta, \varphi)=\alpha \beta \int_{0}^{\frac{G(x ; \varphi)}{1-G(x ; \varphi)}} t^{\alpha-1}(\beta+t)^{-\alpha-1} d t=\frac{G(x ; \varphi)^{\alpha}}{[1-G(x ; \varphi)]^{\alpha}}\left[\beta+\frac{G(x ; \varphi)}{1-G(x ; \varphi)}\right]^{-\alpha}
$$

and

$$
f(x ; \alpha, \beta, \varphi)=\frac{\alpha \beta g(x ; \varphi) G(x ; \varphi)^{\alpha-1}}{[1-G(x ; \varphi)]^{\alpha+1}}\left[\beta+\frac{G(x ; \varphi)}{1-G(x ; \varphi)}\right]^{-\alpha-1},
$$

respectively, where $\alpha>0$ and $\beta>0$ are two additional shape parameters and $g(x ; \varphi)=\mathrm{dG}(x ; \varphi) / \mathrm{d} x$. Henceforth, a random variable (rv) with $\operatorname{PDF}(1.2)$ is denoted by $X \sim \operatorname{OIP}-\mathrm{G}(\alpha, \beta, \varphi)$. For $\beta=1$, the OIP-G family reduces to the exponentiated-G (Exp-G) family (Gupta et al., [17]) . For $\alpha=1$, the OIP-G family becomes the Marshall-Olkin-G (MO-G) class (Marshall and Olkin, [24]). Clearly, for $\alpha=\beta=1$, we obtain the baseline model.

The PDF (1.2) can also be expressed as follows

$$
f(x ; \alpha, \beta, \varphi)=\alpha \beta g(x ; \varphi) G(x ; \varphi)^{\alpha-1}\left[\beta+(1-\beta) G\left(x_{i} ; \varphi\right)\right]^{-\alpha-1} .
$$

The hazard rate function (HRF) of the OIP-G class reduces to

$$
\tau(x ; \alpha, \beta, \varphi)=\frac{\alpha \beta g(x ; \varphi) G(x ; \varphi)^{\alpha-1}\left[\beta+(1-\beta) G\left(x_{i} ; \varphi\right)\right]^{-1}}{\left[\beta+(1-\beta) G\left(x_{i} ; \varphi\right)\right]^{\alpha}-G(x ; \varphi)^{\alpha}} .
$$

The basic motivations for the OIP-G family are the following.

- The special models of the OIP-G family can provide symmetric, left-skewed, right-skewed, reversedJ shaped, unimodal or bimodal shapes for the density function, see left panels in Figures 1-4.

- Its special models accommodate all important hazard rate shapes including constant (CFR), decreasing (DFR), increasing (IFR), decreasing-increasing, upside down bathtub (UBT) or bathtub (BT) failure rate shapes, see right panels in Figures 1-2.

- The OIP-G class generalizes the Exp-G (Gupta et al., [17]) and MO-G (Marshall and Olkin, [24]) families.

- The special models of this family provide consistently better fits than other generated models under the same underlying distribution, see Section 5. 
The rest of the paper is organized as follows. In Section 2, we define two special models of the OIP-G family. In Section 3, we derive some general properties of the OIP-G class. Estimation of the model parameters by maximum likelihood and the observed information matrix are presented in Section 4 . In Section 5, we prove empirically the flexibility and usefulness of the special models of this family using four applications to real data. Finally, some conclusions are given in Section 6.

\section{The OIP-G special models}

This section provides two special models of the OIP-G family. The PDF (1.2) will be most tractable when the functions $G(x)$ and $g(x)$ have simple analytic expressions.

\subsection{The OIP-Lindley (OIPLi) distribution}

The PDF and CDF of the Lindley (Li) distribution with parameter $a>0$ are given by $g(x)=\frac{a^{2}}{1+a}(1+$ $x) \exp (-a x)$ and $G(x)=1-\frac{1+a+a x}{1+a} \exp (-a x), x>0$, respectively. Then, the OIPLi PDF follows from (1.2) as

$$
f(x)=\frac{\alpha \beta a^{2}}{1+a} \frac{(1+x) \exp (-a x)\left[1-\frac{1+a+a x}{1+a} \exp (-a x)\right]^{\alpha-1}}{\left[1-(1-\beta) \frac{1+a+a x}{1+a} \exp (-a x)\right]^{\alpha+1}} .
$$

The special cases of the OIPLi distribution are: the exponentiated or generalized Li (GLi) distribution (Nadarajah et al., [26]) for $\beta=1$; the Marshall-Olkin Li distribution (Ghitany et al., [16]) for $\alpha=1$; and the Li distribution (Lindley, [23]) for $\alpha=\beta=1$. Some possible shapes of the PDF and HRF of the OIPLi model are displayed in Figure 1. These plots reveal great flexibility of the OIPLi density which can be reversed J-shape, concave down, symmetric, left skewed, right skewed, unimodal or bimodal shapes. It is also noted that the HRF of the OIPLi model can be reversed J-shape, CFR, DFR, IFR or BT failure rate shapes.
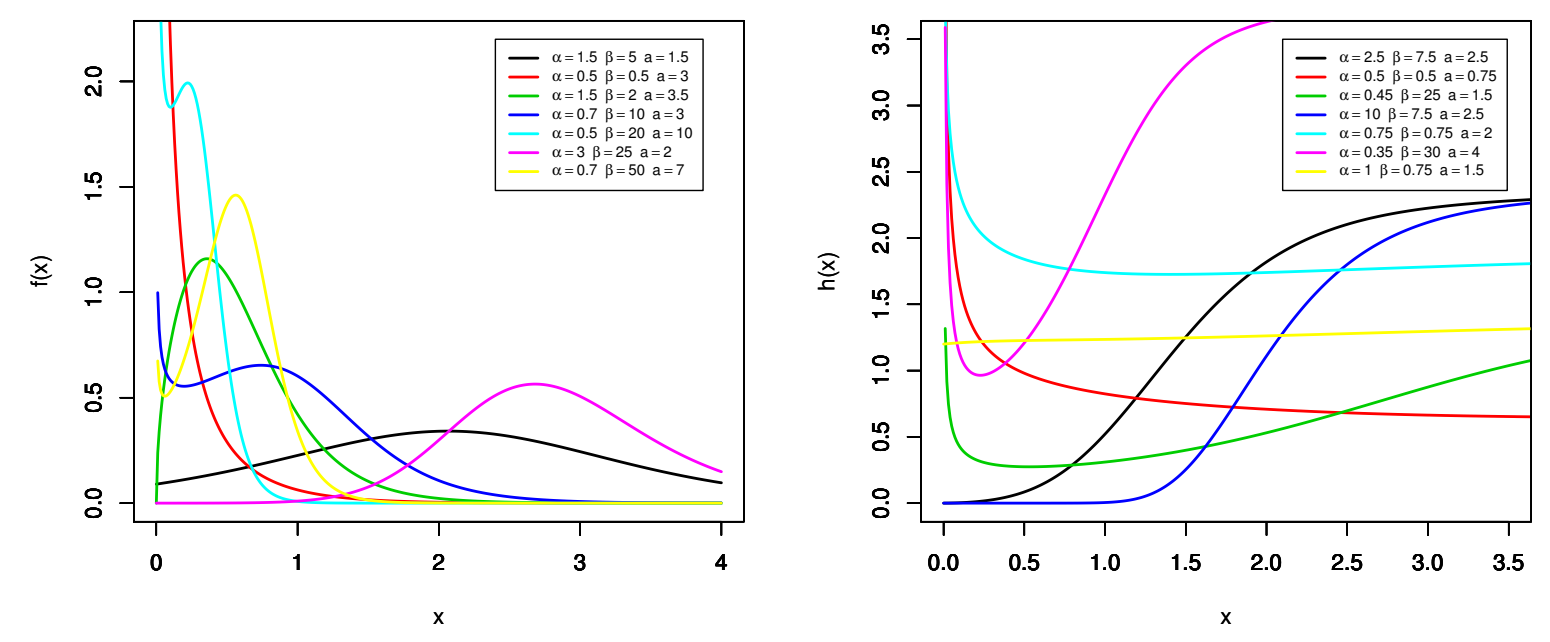

Figure 1: PDF and HRF plots of the OIPLi distribution for selected parameter values.

\subsection{The OIP-Lomax (OIPLx) distribution}

The CDF (for $x>0$ ) of the Lomax (Lx) distribution with positive parameters $a$ and $b$ isG $(x)=$ 
$1-[1+(x / b)]^{-a}$. Then, the OIPLx density function becomes

$$
f(x)=\frac{\alpha \beta a\left[1+\left(\frac{x}{b}\right)\right]^{-(a+1)}\left\{1-\left[1+\left(\frac{x}{b}\right)\right]^{-a}\right\}^{\alpha-1}}{b\left\{1-(1-\beta)\left[1+\left(\frac{x}{b}\right)\right]^{-a}\right\}^{\alpha+1}} .
$$

The special cases of the OIPLi distribution are: the exponentiated Lx (ELx) distribution (Abdul-Moniem and Abdel-Hameed, [1]) for $\beta=1$; the the Marshall-Olkin Lx distribution (Ghitany et al., [15]) for $\alpha=1$; and the Lx distribution for $\alpha=\beta=1$. Plots of the PDF and HRF of the OIPLx model are displayed in Figure 4. It is noted, from Figure 2, that the PDF of the OIPLi model can be unimodal, reversed J-shape, symmetric, left skewed or right skewed. The OIPLx HRF can be J-shape, reversed J-shape, IFR or UBT failure rate shapes.
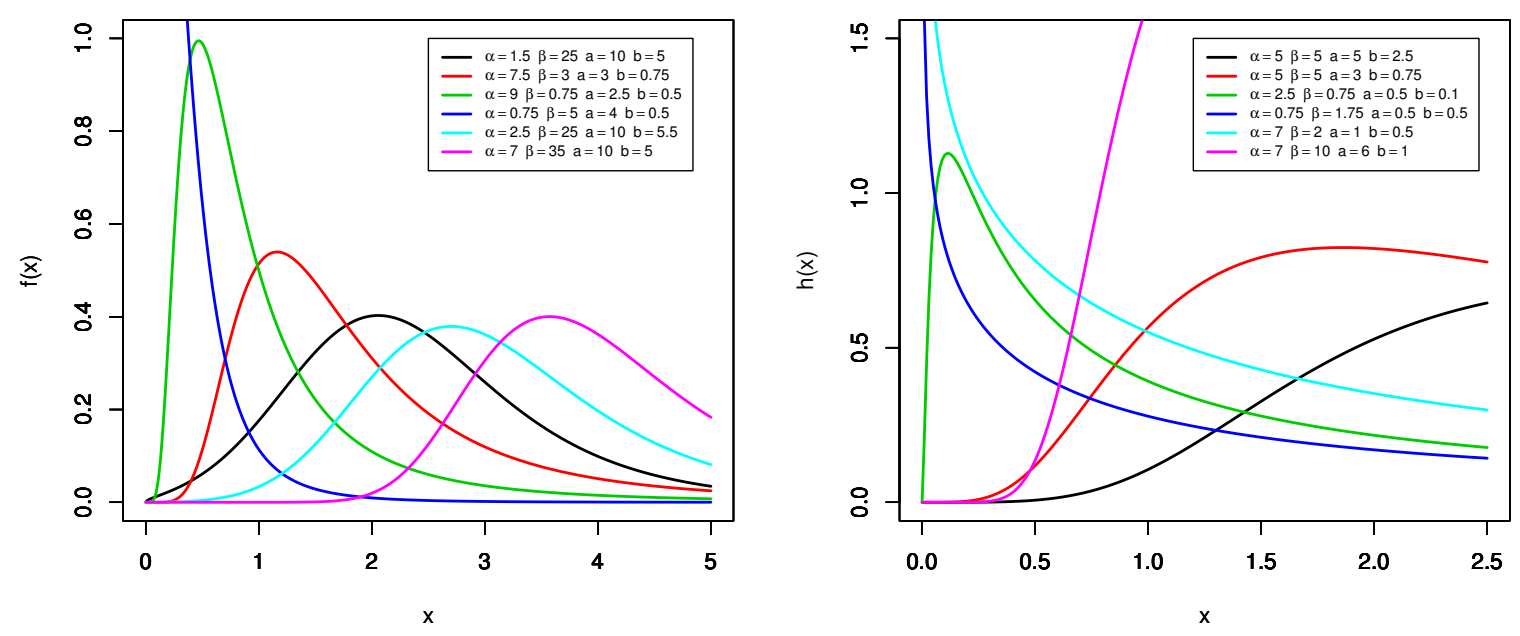

Figure 2: PDF and HRF plots of the OIPLx distribution for selected parameter values.

\section{Properties of the OIP-G class}

In this section, some general properties of the OIP-G family including the linear representation for its PDF, quantile function (QF), moment generating function (MGF), ordinary and incomplete moments, order statistics, probability weighted moments (PWMs), and Rényi entropy are derived.

\subsection{Linear representation}

This section provides a useful linear representation for the PDF of the OIP-G class. The OIP-G PDF can be expressed as

$$
f(x)=\frac{\alpha \beta g(x) G(x)^{\alpha-1}}{[1-G(x)]^{\alpha+1}}\left[\beta+\frac{G(x)}{1-G(x)}\right]^{-\alpha-1} .
$$

Consider the power series

$$
(1+d z)^{-q}=\sum_{n=0}^{\infty}\left(\begin{array}{c}
-q \\
n
\end{array}\right) d^{n} z^{n}
$$

Applying (3.2) to the last term and inserting the resulting expansion in equation (3.1) gives

$$
f(x)=\alpha g(x) \sum_{k=0}^{\infty}\left(\begin{array}{c}
-\alpha-1 \\
k
\end{array}\right) \beta^{-k-\alpha} G(x)^{k+\alpha-1}[1-G(x)]^{-k-\alpha-1} .
$$


For $|z|<1$, the power series holds

$$
(1-z)^{-\mathrm{q}}=\sum_{n=0}^{\infty}(-1)^{\mathrm{n}}\left(\begin{array}{c}
-\mathrm{q} \\
\mathrm{n}
\end{array}\right) z^{\mathrm{n}}
$$

Applying the power series (3.4) to $[1-\mathrm{G}(\mathrm{x})]^{-\mathrm{k}-\alpha-1}$ and substituting in (3.3) gives

$$
f(x)=\alpha \sum_{k, j=0}^{\infty}(-1)^{j}\left(\begin{array}{c}
-\alpha-1 \\
k
\end{array}\right)\left(\begin{array}{c}
-k-\alpha-1 \\
j
\end{array}\right) \beta^{-k-\alpha} g(x) G(x)^{k+j+\alpha-1} .
$$

Or equivalently, one can write

$$
f(x)=\sum_{k, j=0}^{\infty} d_{k, j} h_{k+j+\alpha}(x),
$$

where

$$
d_{k, j}=\frac{\alpha(-1)^{j}}{(k+j+\alpha) \beta^{k+\alpha}}\left(\begin{array}{c}
-\alpha-1 \\
k
\end{array}\right)\left(\begin{array}{c}
-k-\alpha-1 \\
j
\end{array}\right),
$$

and $h_{\delta}(x)=\delta g(x) G(x)^{\delta-1}$ denotes the Exp-G density with power parameter $\delta>0$. Then, the PDF of the OIP-G class can be expressed as a linear combination of Exp-G densities. Thus, several mathematical properties of the OIP-G family can be obtained from those properties of the Exp-G class.

3.2. Quantile and moment generating functions

By inverting (1.1), the QF of $X$ follows as

$$
\mathrm{Q}(\mathrm{u})=\mathrm{Q}_{\mathrm{G}}\left[\frac{\beta u^{\frac{1}{\alpha}}}{1-(1-\beta) \mathrm{u}^{\frac{1}{\alpha}}}\right],
$$

where $Q_{G}(u)=G^{-1}(u)$ is the $Q F$ of the baseline $G$ distribution and $u \in(0,1)$.

The MGF of $X$ follows from equation (3.5) as

$$
M(t)=\sum_{k, j=0}^{\infty} d_{k, j} M_{k+j+\alpha}(t)=\sum_{k, j=0}^{\infty}(k+j+\alpha) d_{k, j} \tau(t, k+j+\alpha-1),
$$

where $M_{k+j+\alpha}(t)$ is the MGF of $Z_{k+j+\alpha}$ (where $Z_{k+j+\alpha}$ is a rv having the Exp-G distribution with power parameter $k+j+\alpha)$ and $\tau(t, k+j+\alpha-1)=\int_{0}^{1} \exp \left[t Q_{G}(u)\right] u^{k+j+\alpha-1} d u$. Then, the MGF of $X$ can be derived from the Exp-G MGF.

\subsection{Ordinary and incomplete moments}

The $r^{\text {th }}$ moment of $X$ follows from (3.5) as

$$
\mu_{r}^{\prime}=E\left(X^{r}\right)=\sum_{k, j=0}^{\infty} d_{k, j} E\left(Z_{k+j+\alpha}^{r}\right) .
$$

The $s^{\text {th }}$ incomplete moment of $X$ can be derived from (3.5) as

$$
m_{s}(z)=\int_{-\infty}^{z} x^{s} f(x) d x=\sum_{k, j=0}^{\infty} d_{k, j} \int_{-\infty}^{z} x^{s} h_{k+j+\alpha}(x) d x .
$$

The first incomplete moment of $X$ can be obtained from (3.6) as

$$
\mathrm{m}_{1}(z)=\sum_{k, j=0}^{\infty} \mathrm{d}_{k, j} \mathrm{~J}_{k+j+\alpha}(z),
$$

where $J_{k+j+\alpha}(z)=\int_{-\infty}^{z} x h_{k+j+\alpha}(x) d x=(k+j+\alpha) \int_{0}^{G(z)} Q_{G}(u) u^{k+j+\alpha-1} d u$ is the first incomplete moment of the Exp-G distribution. It can be computed analytically or numerically from the baseline QF $\mathrm{Q}_{\mathrm{G}}(\mathrm{u})=\mathrm{G}^{-1}(\mathrm{u} ; \phi)$. 
The first incomplete moment can be used to construct Bonferroni and Lorenz curves, which are useful in economics, reliability, demography, insurance and medicine, and mean deviations.

\subsection{Order statistics}

Order statistics are important in several areas of statistical theory and practice. Let $X_{1}, \ldots, X_{n}$ be a random sample from the OIP-G family. The PDF of $X_{i: n}$ can be written as

$$
f_{i: n}(x)=\frac{f(x)}{B(i, n-i+1)} \sum_{j=0}^{n-i}(-1)^{j}\left(\begin{array}{c}
n-i \\
j
\end{array}\right) F(x)^{j+i-1}
$$

where $B(\cdot, \cdot)$ is the beta function. Based on equations (1.1) and (1.2), we can write

$$
f(x) F(x)^{j+i-1}=\frac{\alpha \beta g(x) G(x)^{\alpha(j+i)-1}}{[1-G(x)]^{\alpha(j+i)+1}}\left[\beta+\frac{G(x)}{1-G(x)}\right]^{-\alpha(j+i)-1} .
$$

Based on the same steps in Section 3, one can obtain

$$
\begin{aligned}
f(x) F(x)^{j+i-1}= & \alpha \sum_{k, l=0}^{\infty}(-1)^{l} \beta^{-k-\alpha(j+i)}\left(\begin{array}{c}
-\alpha(j+i)-1 \\
k
\end{array}\right) \\
& \times\left(\begin{array}{c}
-k-\alpha(j+i)-1 \\
l
\end{array}\right) g(x) G(x)^{k+l+\alpha(j+i)-1} .
\end{aligned}
$$

Inserting equation (3.8) in (3.7), the PDF of $X_{i: n}$ follows as

$$
f_{i: n}(x)=\sum_{k, l=0}^{\infty} m_{k, l} h_{k+l+\alpha(j+i)}(x),
$$

where $h_{k+l+\alpha(j+i)}(x)$ is the Exp-G density with power parameter $k+l+\alpha(j+i)$ and

$$
m_{k, l}=\sum_{j=0}^{n-i} \frac{(-1)^{j+l} \alpha \beta^{-k-\alpha(j+i)}}{[k+l+\alpha(j+i)] B(i, n-i+1)}\left(\begin{array}{c}
n-i \\
j
\end{array}\right)\left(\begin{array}{c}
-\alpha(j+i)-1 \\
k
\end{array}\right)\left(\begin{array}{c}
-k-\alpha(j+i)-1 \\
l
\end{array}\right) .
$$

Hence, the PDF of the OIP-G order statistics is a linear combination of Exp-G densities. Based on equation (3.9), the properties of $X_{i: n}$ can be derived from those properties of $Z_{k+l+\alpha(j+i)}$.

\subsection{Probability weighted moments}

For $j, i=1,2, \ldots$, the $(j, i)^{\text {th }}$ PWMs of $X$ is defined by

$$
\rho_{j, i}=E\left\{X^{j} F(X)^{i}\right\}=\int_{-\infty}^{\infty} x^{j} F(x)^{i} f(x) d x .
$$

Based on Equation (3.8), one can write

$$
f(x) F(x)^{i}=\alpha \sum_{k, l=0}^{\infty}(-1)^{l} \beta^{-k-\alpha(i+1)}\left(\begin{array}{c}
-\alpha(i+1)-1 \\
k
\end{array}\right)\left(\begin{array}{c}
-k-\alpha(i+1)-1 \\
l
\end{array}\right) g(x) G(x)^{k+l+\alpha(i+1)-1} .
$$

The above equation can be rewritten as follows

$$
f(x) F(x)^{i}=\sum_{k, l=0}^{\infty} m_{k, l}^{(i)} h_{k+l+\alpha(i+1)}(x),
$$


where

$$
m_{k, l}^{(i)}=\frac{(-1)^{l} \alpha \beta^{-k-\alpha(i+1)}}{[k+l+\alpha(i+1)]}\left(\begin{array}{c}
-\alpha(i+1)-1 \\
k
\end{array}\right)\left(\begin{array}{c}
-k-\alpha(i+1)-1 \\
l
\end{array}\right) .
$$

Then, the PWMs of the OIP-G class is given by

$$
\rho_{j, i}=\sum_{k, l=0}^{\infty} m_{k, l}^{(i)} \int_{-\infty}^{\infty} x^{j} h_{k+l+\alpha(i+1)}(x) d x=\sum_{k, l=0}^{\infty} m_{k, l}^{(i)} E\left(Z_{k+l+\alpha(i+1)}^{j}\right) .
$$

\subsection{Rényi entropy}

The Rényi entropy of a $\mathrm{rv} X$ represents a measure of variation of the uncertainty. It is defined (for $\theta>0$ and $\theta \neq 1$ ) by

$$
I_{\theta}(X)=\frac{1}{1-\theta} \log \left(\int_{-\infty}^{\infty} f(x)^{\theta} d x\right) .
$$

Using the PDF (1.2), we can write

$$
f(x)^{\theta}=\frac{(\alpha \beta)^{\theta} g(x)^{\theta} G(x)^{\theta(\alpha-1)}}{[1-G(x)]^{\theta(\alpha+1)}}\left[\beta+\frac{G(x)}{1-G(x)}\right]^{-\theta(\alpha+1)} .
$$

After applying the power series (3.2) to $\left[\beta+\frac{G(x)}{1-G(x)}\right]^{-\theta(\alpha+1)}$, we obtain

$$
f(x)^{\theta}=\alpha^{\theta} \sum_{k=0}^{\infty}\left(\begin{array}{c}
-\theta(\alpha+1) \\
k
\end{array}\right) \beta^{-\alpha \theta-k} g(x)^{\theta} G(x)^{k+\theta(\alpha-1)}[1-G(x)]^{-k-\theta(\alpha+1)} .
$$

Applying the power series (3.4) to $[1-G(x)]^{-k-\theta(\alpha+1)}$, the last equation reduces to

$$
f(x)^{\theta}=\sum_{k, j=0}^{\infty} \frac{(-1)^{j} \alpha^{\theta}}{\beta^{\alpha \theta+k}}\left(\begin{array}{c}
-\theta(\alpha+1) \\
k
\end{array}\right)\left(\begin{array}{c}
-k-\theta(\alpha+1) \\
j
\end{array}\right) g(x)^{\theta} G(x)^{k+j+\theta(\alpha-1)} .
$$

Then, the Rényi entropy of the OIP-G family reduces to

$$
I_{\theta}(X)=\frac{1}{1-\theta} \log \left[\sum_{k, j=0}^{\infty} t_{k, j} \int_{-\infty}^{\infty} g(x)^{\theta} G(x)^{k+j+\theta(\alpha-1)} d x\right],
$$

where

$$
t_{k, j}=\frac{(-1)^{j} \alpha^{\theta}}{\beta^{\alpha \theta+k}}\left(\begin{array}{c}
-\theta(\alpha+1) \\
k
\end{array}\right)\left(\begin{array}{c}
-k-\theta(\alpha+1) \\
j
\end{array}\right) .
$$

\section{Maximum likelihood estimation}

In this section, we consider the estimation of the unknown parameters of the OIP-G family from complete samples only by the maximum likelihood method. Let $x_{1}, \ldots, x_{n}$ be a random sample from this family with parameters $\alpha, \beta$ and $\varphi$. Let $\theta=\left(\alpha, \beta, \varphi^{\top}\right)^{\top}$ be the $p \times 1$ parameter vector. The log-likelihood function for $\theta$ is given by

$$
\ell=\ell(\theta)=n \log (\alpha \beta)+\sum_{i=1}^{n} \log g\left(x_{i} ; \varphi\right)+(\alpha-1) \sum_{i=1}^{n} \log G\left(x_{i} ; \varphi\right)-(\alpha+1) \sum_{i=1}^{n} \log \left[\beta+(1-\beta) G\left(x_{i} ; \varphi\right)\right] .
$$

The score vector components, $\mathrm{U}(\theta)=\frac{\partial \ell}{\partial \theta}=\left(\mathrm{U}_{\alpha}, \mathrm{U}_{\beta}, \mathrm{U}_{\varphi_{\mathrm{k}}}\right)^{\top}$, are given by

$$
\mathrm{u}_{\alpha}=\frac{\mathrm{n}}{\alpha}+\sum_{i=1}^{\mathrm{n}} \log \mathrm{G}\left(\mathrm{x}_{i} ; \varphi\right)-\sum_{i=1}^{\mathrm{n}} \log \left[\beta+(1-\beta) \mathrm{G}\left(\mathrm{x}_{i} ; \varphi\right)\right],
$$




$$
\mathrm{u}_{\beta}=\frac{\mathrm{n}}{\beta}-(\alpha+1) \sum_{i=1}^{\mathrm{n}} \frac{1-\mathrm{G}\left(\mathrm{x}_{i} ; \varphi\right)}{\beta+(1-\beta) \mathrm{G}\left(\mathrm{x}_{i} ; \varphi\right)}
$$

and

$$
\mathrm{u}_{\varphi_{\mathrm{k}}}=\sum_{i=1}^{n} \frac{g_{k}^{\prime}\left(x_{i} ; \varphi\right)}{\mathrm{g}\left(\mathrm{x}_{i} ; \varphi\right)}+(\alpha-1) \sum_{i=1}^{n} \frac{\mathrm{G}_{k}^{\prime}\left(x_{i} ; \varphi\right)}{\mathrm{G}\left(x_{i} ; \varphi\right)}-\sum_{i=1}^{n} \frac{(\alpha+1)(1-\beta) \mathrm{G}_{k}^{\prime}\left(x_{i} ; \varphi\right)}{\beta+(1-\beta) \mathrm{G}\left(x_{i} ; \varphi\right)},
$$

where $g_{k}^{\prime}\left(x_{i} ; \varphi\right)=\partial g\left(x_{i} ; \varphi\right) / \partial \varphi_{k}$ and $G_{k}^{\prime}\left(x_{i} ; \varphi\right)=\partial G\left(x_{i} ; \varphi\right) / \partial \varphi_{k}$.

Setting the nonlinear system of equations $\mathrm{U}_{\alpha}=\mathrm{U}_{\beta}=0$ and $\mathrm{U}_{\varphi}=0$ and solving them simultaneously yields the MLE $\widehat{\theta}$. This can be done using nonlinear optimization methods such as the quasi-Newton algorithm to maximize $\ell(\theta)$ numerically. For interval estimation of the parameters, we obtain the $p \times p$ observed information matrix $J(\theta)=\left\{\frac{\partial^{2} \ell}{\partial r \partial s}\right\}$ (for $r, s=\alpha, \beta, \varphi$ ), whose elements are

$$
\begin{aligned}
& \mathrm{u}_{\alpha \alpha}=\frac{-\mathrm{n}}{\alpha^{2}}, \quad \mathrm{u}_{\alpha \beta}=-\sum_{i=1}^{n} \frac{1-\mathrm{G}\left(x_{i} ; \varphi\right)}{\beta+(1-\beta) \mathrm{G}\left(x_{i} ; \varphi\right)}, \quad \mathrm{u}_{\alpha \varphi_{k}}=\sum_{i=1}^{n} \frac{\mathrm{G}_{k}^{\prime}\left(x_{i} ; \varphi\right)}{\mathrm{G}\left(x_{i} ; \varphi\right)}-\sum_{i=1}^{n} \frac{(1-\beta) \mathrm{G}_{k}^{\prime}\left(x_{i} ; \varphi\right)}{\beta+(1-\beta) \mathrm{G}(x ; \varphi)}, \\
& \mathrm{u}_{\beta \beta}=\frac{-\mathrm{n}}{\beta^{2}}+(\alpha+1) \sum_{i=1}^{n} \frac{\left[1-\mathrm{G}\left(x_{i} ; \varphi\right)\right]^{2}}{\left[\beta+(1-\beta) \mathrm{G}\left(x_{i} ; \varphi\right)\right]^{2}}, \quad \mathrm{u}_{\beta \varphi_{k}}=-(\alpha+1) \sum_{i=1}^{n} \frac{\mathrm{G}_{k}^{\prime}\left(x_{i} ; \varphi\right)}{\left[\beta+(1-\beta) \mathrm{G}\left(x_{i} ; \varphi\right)\right]^{2}},
\end{aligned}
$$

and

$$
\begin{aligned}
\mathrm{U}_{\varphi_{\mathrm{k}} \varphi_{\mathrm{r}}}= & \sum_{i=1}^{\mathrm{n}} \frac{1}{\mathrm{~g}\left(\mathrm{x}_{i} ; \varphi\right)^{2}}\left\{\mathrm{~g}\left(\mathrm{x}_{\mathrm{i}} ; \varphi\right) \mathrm{g}_{\mathrm{kr}}^{\prime \prime}\left(x_{i} ; \varphi\right)-\mathrm{g}_{\mathrm{k}}^{\prime}\left(x_{i} ; \varphi\right) \mathrm{g}_{\mathrm{r}}^{\prime}\left(x_{i} ; \varphi\right)\right\} \\
& +\sum_{i=1}^{n} \frac{(\alpha-1)}{\mathrm{G}\left(x_{i} ; \varphi\right)^{2}}\left\{\mathrm{G}\left(x_{i} ; \varphi\right) \mathrm{G}_{\mathrm{kr}}^{\prime \prime}\left(x_{i} ; \varphi\right)-\mathrm{G}_{\mathrm{k}}^{\prime}\left(x_{i} ; \varphi\right) \mathrm{G}_{\mathrm{r}}^{\prime}\left(x_{i} ; \varphi\right)\right\} \\
& -(\alpha+1) \sum_{i=1}^{n} \frac{\left[\frac{\beta}{(1-\beta)}+\mathrm{G}\left(x_{i} ; \varphi\right)\right] \mathrm{G}_{\mathrm{kr}}^{\prime \prime}\left(x_{i} ; \varphi\right)-\mathrm{G}_{\mathrm{k}}^{\prime}\left(x_{i} ; \varphi\right) \mathrm{G}_{\mathrm{r}}^{\prime}\left(x_{i} ; \varphi\right)}{(1-\beta)^{-2}\left[\beta+(1-\beta) \mathrm{G}\left(x_{i} ; \varphi\right)\right]^{2}}
\end{aligned}
$$

where

$$
{\overline{G_{k}}}^{\prime}\left(x_{i} ; \varphi\right)=\partial \bar{G}\left(x_{i} ; \varphi\right) / \partial \varphi_{k}, \quad g_{k r}^{\prime \prime}\left(x_{i} ; \varphi\right)=\partial^{2} g\left(x_{i} ; \varphi\right) / \partial \varphi_{k} \partial \varphi_{r}, \quad \text { and } \quad G_{k r}^{\prime \prime}\left(x_{i} ; \varphi\right)=\partial^{2} G\left(x_{i} ; \varphi\right) / \partial \varphi_{k} \varphi_{r} .
$$

\section{Two applications to real data}

In this section, the importance and flexibility of the OIPLx and OIPLi distributions are illustrated using two real data sets. The fitted models are compared using some goodness-of-fit criteria including the $-\widehat{\ell}$ (where $\widehat{\ell}$ is the maximized log-likelihood), CVM (Cramér-Von Mises), AD (Anderson-Darling), and KS (Kolmogorov Smirnov with its p-value (PV)) statistics.

\subsection{An application of the OIPLx distribution}

The data contain 213 observations on the number of successive failures of the air conditioning system of fleet of 13 Boeing 720 jet airplanes (Proschan, [27]). We shall compare the fits of the OIPLx distribution with some competitive models namely: the Weibull Lomax (WLx) (Tahir et al., [30]), odd exponentiated half-logistic Lomax (OEHLLx) (Afify et al., [2]), Lomax Weibull (LxW) (Cordeiro et al., [13]), Kumaraswamy Lomax (KwLx) (Lemonte and Cordeiro, [22]), exponentiated Lomax (ELx) (Gupta et al., [17]), complementary generalized transmuted Poisson Lomax (CGTPLx) (Alizadeh et al., [4]), complementary geometric transmuted Lomax (CGcTLx) (Afify et al., [3]), and Lomax distributions whose PDFs are given by

$$
\text { WLx: } f(x)=\frac{a b \alpha}{\beta}(1+x / \beta)^{b \alpha-1}\left[1-(1+x / \beta)^{-\alpha}\right]^{b-1} e^{-a\left[(1+x / \beta)^{\alpha}-1\right]^{b}},
$$




$$
\begin{aligned}
& \text { OEHLLx: } f(x)=\frac{2 \alpha \theta a}{b}[1+(x / b)]^{a-1} e^{\frac{-\theta\left\{1-[1+(x / b)]^{-a}\right\}}{[1+(x / b)]^{-a}}} \\
& \times\left\{1-\mathrm{e}^{\frac{-\theta\left\{1-[1+(x / b)]^{-a}\right\}}{[1+(x / b)]^{-a}}}\right\}^{\alpha-1}\left\{1+\mathrm{e}^{\frac{-\theta\left\{1-[1+(x / b)]^{-a}\right\}}{[1+(x / b)]^{-a}}}\right\}^{-\alpha-1}, \\
& \text { LxW: } f(x)=\alpha b \beta^{\alpha} a^{b} x^{b-1}\left[\beta+(a x)^{b}\right]^{-\alpha-1}, \\
& \text { KwLx: } f(x)=\frac{a b \alpha}{\beta}(1+x / \beta)^{-\alpha-1}\left[1-(1+x / \beta)^{-\alpha}\right]^{a-1}\left\{1-\left[1-(1+x / \beta)^{-\alpha}\right]^{a}\right\}^{b-1} \text {, } \\
& \text { ELx: } f(x)=\frac{\alpha a}{b}[1+(x / b)]^{-(a+1)}\left\{1-[1+(x / b)]^{-a}\right\}^{\alpha-1} \text {, } \\
& \text { CGTPLx: } f(x)=\frac{\theta a[1+(x / b)]^{-(a+1)} e^{`[1+(x / b)]^{-a}}}{b\left(e^{`}-1\right)}\left\{1-\lambda \frac{\left(e^{`}+1\right)-2 e^{`[1+(x / b)]^{-a}}}{e^{`}-1}\right\} \text {, } \\
& \text { CGcTLx: } f(x)=\frac{\theta a[1+(x / b)]^{-(a+1)}\left[1-\lambda+2 \lambda[1+(x / b)]^{-a}\right]}{b(1-(1-\theta)(1-[1+(x / b)])\{1+\lambda[1+(x / b)]\})^{2}} \text {. }
\end{aligned}
$$

The parameters of the above densities are all positive real numbers except $|\lambda| \leqslant 1$ for the CGTPLx and CGcTLx distributions, and $\theta \in(0,1)$ for the CGcTLx model.

Table 1 displays the values of goodness-of-fit statistics and MLEs and their SEs for the OIPLx distribution and other competitive models.

The values in this table indicate that the OIPLx distribution has the lowest values for all goodness-of-fit statistics among all fitted distributions. So, it can be chosen as the best model to fit this data set.

The histogram of the data and the fitted distributions are displayed in Figure 3. Figure 4 provides the PP plots for the fitted models. These plots reveal that the OIPLx distribution has a close fit to this data set.

Table 1: The $-\widehat{\ell}, \mathrm{CVM}, \mathrm{AD}, \mathrm{KS}$, and estimates (PV and SEs in parentheses).

\begin{tabular}{|l|c|c|c|c|c|c|c|c|}
\hline Distribution & $-\widehat{l}$ & CVM & AD & KS & \multicolumn{5}{|c|}{ Estimates } \\
\hline OIPLx & 976.838 & 0.0303 & 0.2268 & 0.0421 & 1.5247 & 0.2278 & 176.6445 & 26281.07 \\
$(\alpha, \beta, a, b)$ & & & & $(0.9076)$ & $(0.3158)$ & $(0.1083)$ & $(40.7740)$ & $(1180.07)$ \\
\hline WLx & 977.091 & 0.0331 & 0.2482 & 0.0408 & 0.2699 & 2.5270 & 0.4201 & 2.0172 \\
$(\alpha, \beta, a, b)$ & & & & $(0.9259)$ & $(0.2905)$ & $(8.2042)$ & $(0.7678)$ & $(1.6727)$ \\
\hline OEHLLx & 977.747 & 0.0410 & 0.3013 & 0.0440 & 1.9283 & 0.4192 & 0.4457 & 1.3381 \\
$(\alpha, \lambda, a, b)$ & & & & $(0.8780)$ & $(1.2444)$ & $(0.7451)$ & $(0.0883)$ & $(5.1206)$ \\
\hline LxW & 979.430 & 0.0809 & 0.5400 & 0.0478 & 2.9217 & 13.8280 & 0.0628 & 1.0949 \\
$(\alpha, \beta, a, b)$ & & & & $(0.8060)$ & $(1.9127)$ & $(52.5847)$ & $(0.2151)$ & $(0.1310)$ \\
\hline KwLx & 979.118 & 0.0659 & 0.4566 & 0.0486 & 0.1171 & 110.066 & 1.2056 & 29.5269 \\
$(\alpha, \beta, a, b)$ & & & & $(0.7905)$ & $(0.5458)$ & $(130.510)$ & $(0.2470)$ & $(154.188)$ \\
\hline ELx & 979.021 & 0.0665 & 0.4575 & 0.0488 & 1.2158 & 3.1479 & 171.446 & \\
$(\alpha, a, b)$ & & & & $(0.7868)$ & $(0.2102)$ & $(1.4124)$ & $(119.670)$ & \\
\hline CGTPLx & 979.063 & 0.0834 & 0.5386 & 0.0526 & 0.2649 & 1.0458 & 863.240 & 118093 \\
$(\lambda, \theta, a, b)$ & & & & $(0.7046)$ & $(0.7459)$ & $(1.4994)$ & $(222.764)$ & $(781.107)$ \\
\hline CGcTLx & 979.470 & 0.0967 & 0.6194 & 0.0461 & 0.3660 & 0.9999 & 8.3551 & 812.020 \\
$(\lambda, \theta, a, b)$ & & & & $(0.8406)$ & $(0.4246)$ & $(0.7992)$ & $(20.6326)$ & $(2639.24)$ \\
\hline Lx & 979.722 & 0.0981 & 0.6305 & 0.0456 & 4.9059 & 350.699 & & \\
$(a, b)$ & & & & $(0.8502)$ & $(2.3516)$ & $(199.899)$ & & \\
\hline
\end{tabular}



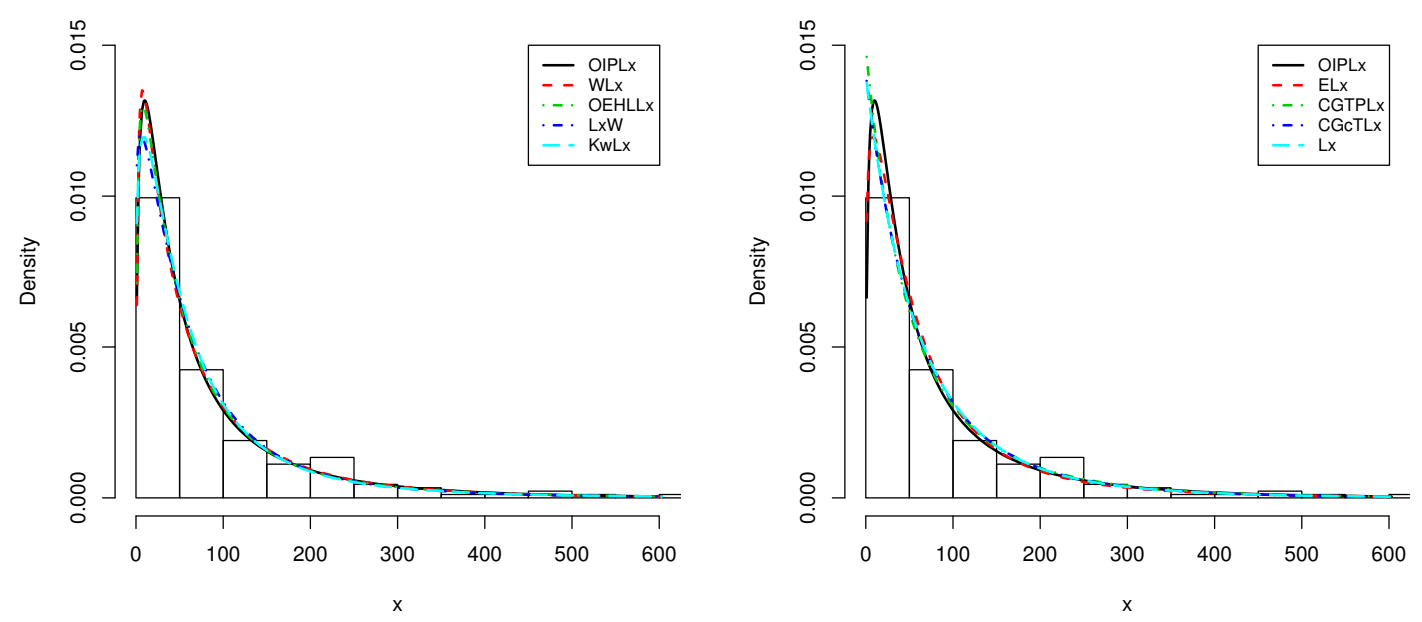

Figure 3: The estimated OIPLx PDF and other estimated PDFs.
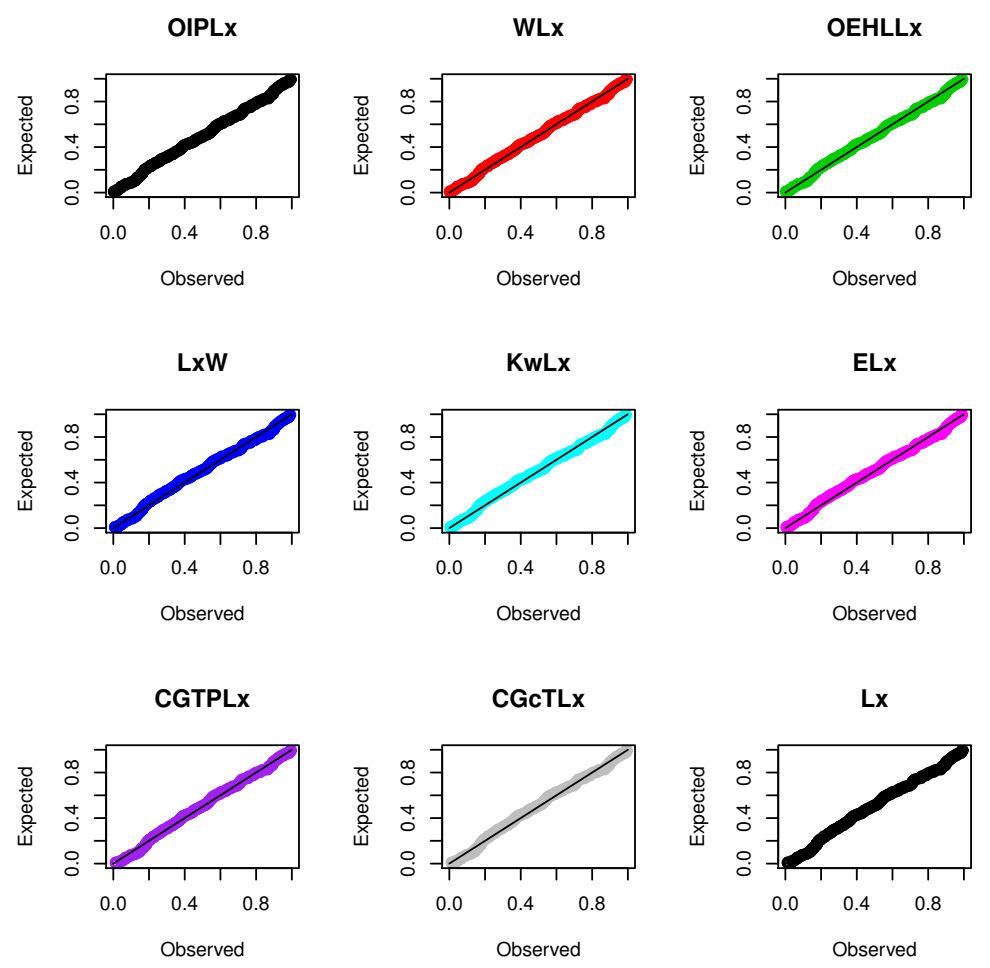

Figure 4: PP plots of the OIPLx distribution and other competitive distributions.

\subsection{An application of the OIPLi distribution}

The data contain 128 observations on the remission times (in months) of bladder cancer patients (Lee and Wang, [21]). We shall compare the fits of the OIPLi distribution with some competitive models namely: the Lindley Weibull (LiW) (Cordeiro et al., [10]), transmuted two-parameter Lindley (TTLi) (Kemaloglu and Yilmaz, [18]), Weibull Lindley (WLi) (Asgharzadeh et al., [7]), Quasi Lindley (QLi ) (Shanker and Mishra, [29]), complementary geometric transmuted Lindley (CGcTLi) (Afify et al., [3]), generalized Lindley (GLi) (Nadarajah et al., [26]), new weighted Lindley (NWLi) (Asgharzadeh et al., [6]), and Lindley 
distributions whose PDFs are given by

LiW: $f(x)=\frac{\beta \theta^{2}}{\theta+1}\left[\alpha^{\beta} x^{\beta-1}+\alpha^{2 \beta} x^{2 \beta-1}\right] \exp \left[-\theta(\alpha x)^{\beta}\right]$

TTLi: $f(x)=\frac{a^{2}}{\alpha+a}(1+\alpha x) \exp (-a x)\left[1-\lambda+2 \lambda \frac{\alpha+a+\alpha a x}{\alpha+a} \exp (-a x)\right]$,

WLi: $f(x)=\frac{\exp \left[-\lambda x-(\beta x)^{\alpha}\right]}{1+\lambda}\left[\alpha \lambda(\beta x)^{\alpha}+\alpha \beta(1+\lambda)(\beta x)^{\alpha-1}+\lambda^{2}(1+x)\right]$,

QLi: $f(x)=\frac{a}{\alpha+1}(\alpha+a x) \exp (-a x)$,

CGcTLi: $f(x)=\frac{\frac{\theta \alpha^{2}}{1+\alpha}(1+x) \exp (-\alpha x)\left[1-\lambda+2 \lambda \frac{1+\alpha+\alpha x}{1+\alpha} \exp (-\alpha x)\right]}{\left\{1-(1-\theta)\left[1-\frac{1+\alpha+\alpha x}{1+\alpha} \exp (-\alpha x)\right]\left[1+\lambda \frac{1+\alpha+\alpha x}{1+\alpha} \exp (-\alpha x)\right]\right\}^{2}}$,

GLi: $f(x)=\frac{\alpha a^{2}}{1+a}(1+x) \exp (-a x)\left[1-\frac{1+a+a x}{1+a} \exp (-a x)\right]^{\alpha-1}$,

NWLi: $f(x)=\frac{a^{2}(1+\alpha)^{2}(1+x)[1-\exp (-\alpha a x)] \exp (-a x)}{a \alpha(1+\alpha)+\alpha(2+\alpha)}$.

The parameters of the above densities are all positive real numbers except $|\lambda| \leqslant 1$ for the TTLi and CGTPLi distributions, $\theta \in(0,1)$ for the CGcTLi model, and $\alpha>-1$ for the QLi model.

Table 2 displays the values of goodness-of-fit statistics and MLEs and their SEs for the OIPLi distribution and other fitted models.

The values in this table indicate that the OIPLi distribution has the lowest values for all goodness-of-fit statistics among all fitted distributions. So, it can be chosen as the best model to fit this data set.

The histogram of the data and the fitted distributions are displayed in Figure 5. Figure 6 provides the PP plots for the fitted models. These plots reveal that the OIPLi distribution has a close fit to this data set.

Table 2: The $-\widehat{\ell}, \mathrm{CVM}, \mathrm{AD}, \mathrm{KS}$, and estimates (PV and SEs in parentheses).

\begin{tabular}{|l|c|c|c|c|c|c|c|}
\hline Distribution & $-\widehat{l}$ & $\mathrm{CVM}$ & $\mathrm{AD}$ & $\mathrm{KS}$ & \multicolumn{3}{|c|}{ Estimates } \\
\hline $\begin{array}{l}\text { OIPLi } \\
(\alpha, \beta, \mathrm{a})\end{array}$ & 409.447 & 0.0160 & 0.1115 & 0.0315 & 1.1017 & 0.0956 & 0.0741 \\
$\mathrm{LiW}$ & & & & $(0.9995)$ & $(0.1988)$ & $(0.0858)$ & $(0.0335)$ \\
$(\alpha, \beta, \theta)$ & 411.666 & 0.0734 & 0.4457 & 0.0572 & 17.6581 & 0.7498 & 0.0460 \\
& & & & $(0.7950)$ & $(25.9839)$ & $(0.0498)$ & $(0.0453)$ \\
\hline $\mathrm{TTLi}$ & 412.941 & 0.1170 & 0.6877 & 0.0636 & 0.1578 & 0.1170 & 0.7128 \\
$(\alpha, \mathrm{a}, \lambda)$ & & & & $(0.6771)$ & $(0.1670)$ & $(0.0293)$ & $(0.2068)$ \\
\hline $\mathrm{WLi}$ & 414.088 & 0.1314 & 0.7867 & 0.0700 & 1.0478 & 0.1045 & 0.0010 \\
$(\alpha, \beta, \lambda)$ & & & & $(0.5568)$ & $(0.0675)$ & $(0.0093)$ & $(0.0176)$ \\
\hline $\mathrm{QLi}$ & 414.343 & 0.1193 & 0.7162 & 0.0846 & 117.887 & 0.1076 & \\
$(\alpha, \mathrm{a})$ & & & & $(0.3185)$ & $(1483.01)$ & $(0.0146)$ & \\
\hline $\mathrm{CGCTLi}$ & 415.163 & 0.1043 & 0.6176 & 0.0898 & 0.1558 & 0.9990 & 0.6169 \\
$(\alpha, \theta, \lambda)$ & & & & $(0.2521)$ & $(0.0203)$ & $(0.2547)$ & $(0.1695)$ \\
\hline $\mathrm{GLi}$ & 416.285 & 0.1920 & 1.1472 & 0.0927 & 0.7336 & 0.1648 & \\
$(\alpha, \mathrm{a})$ & & & & $(0.2204)$ & $(0.0911)$ & $(0.0166)$ & \\
\hline $\mathrm{NWLi}$ & 419.464 & 0.1696 & 1.0127 & 0.1165 & 235.080 & 0.1961 & \\
$(\alpha, \mathrm{a})$ & & & & $(0.0618)$ & $(558.739)$ & $(0.0123)$ & \\
\hline $\mathrm{Li}$ & 419.529 & 0.1717 & 1.0257 & 0.1163 & 0.1960 & & \\
$(\mathrm{a})$ & & & & $(0.0623)$ & $(0.0123)$ & & \\
\hline
\end{tabular}



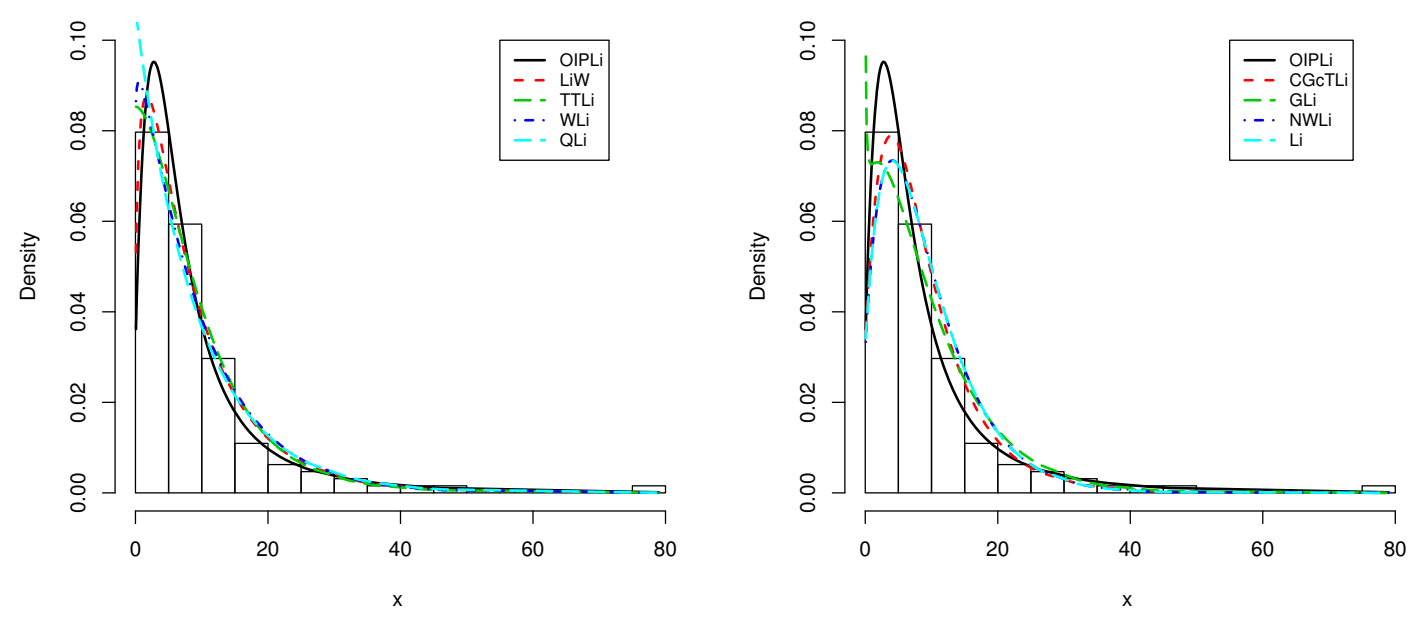

Figure 5: The estimated OIPLi PDF and other estimated PDFs.

OIPLi

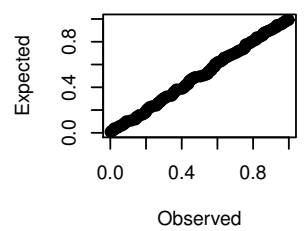

WLi

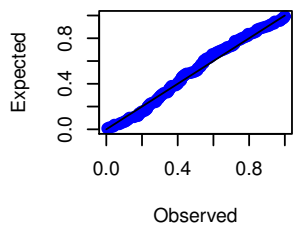

GLi

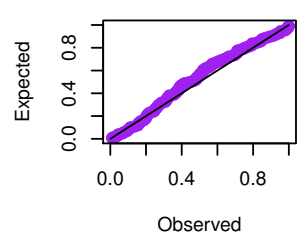

LiW

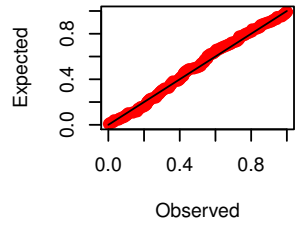

QLi

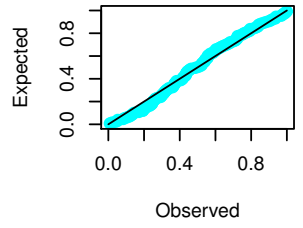

NWLi

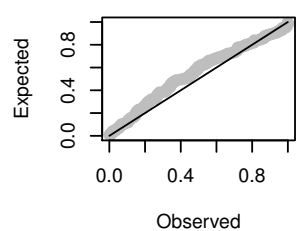

TTLi

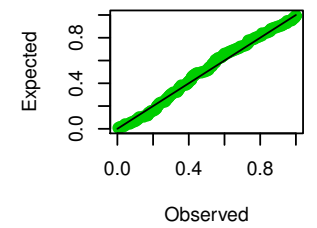

CGCTLi

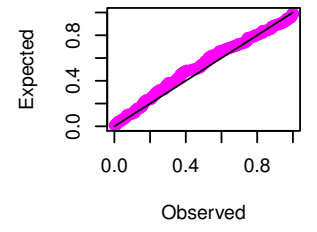

Li

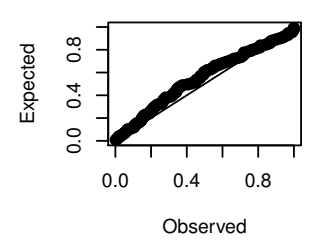

Figure 6: PP plots of the OIPLi distribution and other competitive distributions.

\section{Conclusions}

In this paper, we propose a new family of distributions with two extra shape parameters called the odd inverse Pareto-G (OIP-G) family. The new family extends several widely known distributions and four of its special models are provided. The OIP-G density function is a linear combination of exponentiated-G densities. Some of its mathematical properties are derived. The model parameters are estimated by the method of maximum likelihood. Four real data examples are used to illustrate the flexibility of the special models of the new family in comparing with other well known models in the literature. 


\section{References}

[1] I. B. Abdul-Moniem, H. F. Abdel-Hameed, On exponentiated Lomax distribution, Int. J. Math. Arch., 3 (2012), 21442150. 2.2

[2] A. Z. Afify, E. Altun, M. Alizadeh, G. Ozel, G. G. Hamedani, The odd exponentiated half-logistic-G family: properties, characterizations and applications, Chil. J. Stat., 8 (2017), 65-91. 5.1

[3] A. Z. Afify, G. M. Cordeiro, S. Nadarajah, H. M. Yousof, G. Ozel, Z. M. Nofal, E. Altun, The complementary geometric transmuted-G family of distributions: model, properties and application, Hacet. J. Math. Statist., 47 (2018), $1348-1374$. $5.1,5.2$

[4] M. Alizadeh, H. M. Yousof, A. Z. Afify, G. M. Cordeiro, M. Mansoor, The Complementary Generalized Transmuted Poisson-G Family of Distributions, Austrian J. Statist., 47 (2018), 51-71. 5.1

[5] A. Alzaatreh, C. Lee, F. Famoye, A new method for generating families of distributions, Metron, 71 (2013), 63-79. 1

[6] A. Asgharzadeh, H. S. Bakouch, S. Nadarajah, F. Sharafi, A new weighted Lindley distribution with application, Braz. J. Probab. Stat., 30 (2016), 1-27. 5.2

[7] A. Asgharzadeh, S. Nadarajah, F. Sharafi, Weibull Lindley distribution, REVSTAT, 16 (2018), 87-113. 5.2

[8] M. Bourguignon, R. B. Silva, G. M. Cordeiro, The Weibull-G family of probability distributions, J. Data Sci., 12 (2014), 53-68. 1

[9] G. M. Cordeiro, A. Z. Afify, E. M. M. Ortega, A. K. Suzuki, M. E. Mead, The odd Lomax generator of distributions: Properties, estimation and applications, J. Comput. Appl. Math., 347 (2019), 222-237. 1

[10] G. M. Cordeiro, A. Z. Afify, H. M. Yousof, S. Cakmakyapan, G. Ozel, The Lindley Weibull Distribution: Properties and Applications, Anais da Academia Brasileira de Ciências, 90 (2018), 2579-2598. 5.2

[11] G. M. Cordeiro, A. Z. Afify, H. M. Yousof, R. R. Pescim, G. R. Aryal, The exponentiated Weibull-H family of distributions: Theory and Applications, Mediterr. J. Math., 14 (2017), 22 pages. 1

[12] G. M. Cordeiro, M. de Castro, A new family of generalized distributions, J. Stat. Comput. Simul., 81 (2011), $883-898$.

[13] G. M. Cordeiro, E. M. M. Ortega, B. V. Popović, R. R. Pescim, The Lomax generator of distributions: properties, minification process and regression model, Appl. Math. Comput., 247 (2014), 465-486. 1, 5.1

[14] N. Eugene, C. Lee, F. Famoye, Beta-normal distribution and its applications, Comm. Statist. Theory Methods, 31 (2002), 497-512. 1

[15] M. E. Ghitany, F. A. Al-Awadhi, L. A. Alkhalfan, Marshall-Olkin extended Lomax distribution and its application to censored data, Comm. Statist. Theory Methods, 36 (2007), 1855-1866. 2.2

[16] M. E. Ghitany, D. K. Al-Mutairi, F. A. Al-Awadhi, M. M. Al-Burais, Marshall-Olkin extended Lindley distribution and its application, Int. J. Appl. Math., 25 (2012), 709-721. 2.1

[17] R. C. Gupta, P. L. Gupta, R. D. Gupta, Modeling failure time data by Lehman alternatives, Comm. Statist. Theory Methods, 27 (1998), 887-904. 1, 5.1

[18] S. A. Kemaloglu, M. Yilmaz, Transmuted two-parameter Lindley distribution, Comm. Statist. Theory Methods, 46 (2017), 11866-11879. 5.2

[19] S. A. Klugman, H. H. Panjer, G. E. Willmot, Loss models: from data to decisions, John Wiley \& Sons, Hoboken, (2012). 1

[20] D. Kundu, M. Z. Raqab, Generalized Rayleigh distribution: different methods of estimations, Comput. Statist. Data Anal., 49 (2005), 187-200.

[21] E. T. Lee, J. W. Wang, Statistical methods for survival data analysis: Third edition, John Wiley \& Sons, Hoboken, (2003). 5.2

[22] A. J. Lemonte, G. M. Cordeiro, An extended Lomax distribution, Statistics, 47 (2013), 800-816. 5.1

[23] D. V. Lindley, Fiducial distributions and Bayes' theorem, J. Roy. Statist. Soc. Ser. B, 20 (1958), 102-107. 2.1

[24] A. W. Marshall, I. Olkin, A new method for adding a parameter to a family of distributions with application to the exponential and Weibull families, Biometrika, 84 (1997), 641-652. 1

[25] G. S. Mudholkar, D. K. Srivastava, Exponentiated Weibull family for analyzing bathtub failure-real data, IEEE Trans. Reliab., 42 (1993), 299-302.

[26] S. Nadarajah, H. S. Bakouch, R. Tahmasbi, A generalized Lindley distribution, Sankhya B, 73 (2011), 331-359. 2.1, 5.2

[27] F. Proschan, Theoretical explanation of observed decreasing failure rate, Technometrics, 5 (1963), 375-383. 5.1

[28] J. W. S. Rayleigh, On the resultant of a large number of vibration of the same pitch and arbitrary phase, Philosophical Magazine: Series 5, 43 (1880), 259-272.

[29] R. Shanker, A. Mishra, A quasi Lindley distribution, African J. Math. Comput. Sci. Res., 6 (2013), 64-71. 5.2

[30] M. H. Tahir, G. M. Cordeiro, M. Mansoor, M. Zubair, The Weibull-Lomax distribution: properties and applications, Hacet. J. Math. Stat., 44 (2015), 455-474. 5.1

[31] H. Torabi, N. H. Montazari, The gamma-uniform distribution and its application, Kybernetika (Prague), 48 (2012), 16-30. 1

[32] W. Weibull, A statistical distribution function of wide applicability, J. Appl. Mech., 18 (1951), 290-293.

[33] K. Zografos, N. Balakrishnan, On families of beta and generalized gamma generated distributions and associated inference, Stat. Methodol., 6 (2009), 344-362. 1 